\title{
Reduced soluble receptor for advanced glycation end-products in COPD
}

\author{
D.J. Smith*,+, S.T. Yerkovich",+, M.A. Towers*,\#, M.L. Carroll\#, \\ R. Thomas ${ }^{*}$ and J.W. Upham*,
}

ABSTRACT: The soluble receptor for advanced glycation end-products (sRAGE) has antiinflammatory properties, and deficiency of circulating sRAGE is associated with various human diseases. Whether SRAGE concentrations are reduced in chronic obstructive pulmonary disease (COPD) has not been determined. The aim of this study was to determine plasma levels of SRAGE in COPD patients and establish whether SRAGE varies in relation to forced expiratory volume in $1 \mathrm{~s}$ (FEV1) and other inflammatory markers.

61 COPD patients and 42 healthy controls were recruited. Plasma sRAGE, C-reactive protein (CRP) and serum amyloid A (SAA) were measured in patients with stable COPD. A subgroup had measurements during acute exacerbations of COPD (AECOPD).

sRAGE was significantly lower in stable COPD than in healthy controls $(p<0.001)$, while CRP $(p<0.001)$ and SAA $(p=0.015)$ were higher in stable COPD than in healthy controls. Multiple linear regression confirmed that COPD was negatively associated with sRAGE $(p<0.001)$. Plasma SRAGE was positively correlated with FEV $1\left(r^{2}=0.530, p<0.001\right)$, while CRP and SAA were inversely proportional to FEV1. Multiple linear regression analysis showed that only sRAGE was a strong predictor of FEV1. AECOPD were associated with even lower sRAGE levels that increased with convalescence.

Circulating SRAGE is lower in COPD and shows a strong correlation to the degree of airflow limitation.

KEYWORDS: Biomarker, chronic obstructive pulmonary disease, inflammation, soluble receptor for advanced glycation end-products

hronic obstructive pulmonary disease (COPD) is closely linked to the inhalation of noxious agents, especially cigarette smoke, and is an increasing cause of morbidity and mortality throughout the world [1, 2]. While COPD is predominantly a disease of the lungs, there is growing awareness that COPD is associated with clinically relevant, extra-pulmonary complications including atherosclerosis, osteoporosis and skeletal muscle wasting [3]. Some of these complications of COPD are undoubtedly driven by shared risk factors such as smoking, though systemic inflammation may also play an important role [3-5].

There has, therefore, been considerable interest in identifying new molecules in the blood that distinguish COPD patients from healthy control subjects. Such biomarkers might provide novel insight into inflammatory pathways in COPD, and have the potential to predict disease progression and the risk of comorbidities. Several studies have reported increased blood concentrations of Creactive protein $(\mathrm{CRP})$, fibrinogen, inflammatory cytokines and other novel biomarkers in stable COPD, relative to healthy control subjects $[4,6]$.

The receptor for advanced glycation end-products (RAGE) mediates responses to cell injury induced by a variety of stimuli including hyperglycaemia, oxidative stress, ageing and hypoxia $[7,8]$. The ligands for RAGE include not only advanced glycation end-products, but also serum amyloid A (SAA) and members of the S100 protein family, and when these ligands bind RAGE, a pro-inflammatory cascade is initiated. RAGE has been implicated in the complications of diabetes, as well as atherosclerosis and rheumatoid arthritis [7-9]. RAGE has several isoforms including a soluble form known as sRAGE that appears to acts as a decoy receptor, binding RAGE ligands in the extracellular fluid
AFFILIATIONS

*Dept of Respiratory Medicine,

Princess Alexandra Hospital,

\# School of Medicine, The University

of Queensland, and

"Diamantina Institute, The University

of Queensland, Brisbane, Australia.

${ }^{+}$Both authors contributed equally to

this study.

CORRESPONDENCE

J.W. Upham

The University of Queensland School of Medicine

Princess Alexandra Hospital

Ipswich Road

Woolloongabba

Brisbane

QId 4102

Australia

E-mail: j.upham@uq.edu.au

Received:

Feb 222010

Accepted after revision:

June 152010

First published online:

July 012010 
and thereby protecting against inflammation and tissue injury. Treatment with sRAGE can successfully reverse inflammation in a variety of animal models $[9,10]$ while in humans, coronary artery disease [11] and rheumatoid arthritis [12] are both associated with lower blood levels of sRAGE relative to healthy control subjects. It has therefore been proposed that decreased circulating sRAGE may be a marker of deficient inflammatory control [12].

Both RAGE and sRAGE are constitutively expressed in normal lung tissue [13-15], though their functional roles have not been clearly defined. Acute lung injury is associated with increased concentrations of RAGE in bronchoalveolar lavage (BAL) fluid and plasma $[16,17]$. In contrast, membrane RAGE and sRAGE are reduced in patients with idiopathic pulmonary fibrosis [14, 18], and RAGE may have a protective role in animal models of pulmonary fibrosis [14, 18]. A recent study has shown that membrane RAGE is overexpressed in the airways of COPD patients, though circulating sRAGE was not measured in that study [19]. Circulating concentrations of the RAGE ligand SAA are elevated during acute COPD exacerbations [20].

We hypothesised that plasma sRAGE would be reduced in patients with stable COPD, relative to healthy control subjects. The study also examined whether plasma sRAGE varied in relation to the extent of airflow obstruction, and to the concentrations of CRP and SAA. In a subset of COPD patients, sRAGE was measured during acute exacerbations and later during convalescence.

\section{METHODS}

\section{Study subjects}

Patients met joint European Respiratory Society (ERS)/ American Thoracic Society (ATS) criteria for a clinical definition of COPD [21]. All were current or former smokers with fixed airflow obstruction, forced expiratory volume in $1 \mathrm{~s}$ (FEV1)/forced vital capacity (FVC) ratio of $<0.7$ and postbronchodilator FEV1 $<80 \%$ predicted. Severity of COPD was graded as per the global initiative for chronic obstructive lung disease (GOLD) criteria [2]. Exclusion criteria included coexistent pulmonary disease (e.g. interstitial pulmonary fibrosis, bronchiectasis or granulomatous lung disease), malignancy, long-term oral corticosteroid therapy and renal impairment [22]. Patients with cardiac disease and diabetes mellitus were not excluded.

Cases with "stable" COPD were recruited from the outpatient clinic at a single tertiary referral hospital, while cases with an "acute" exacerbation of COPD (AECOPD) were recruited within $48 \mathrm{~h}$ of hospital admission. Control subjects (both nonsmokers and smokers) were spouses or partners of COPD cases, or were recruited by advertisement. All control subjects had no prior or current symptoms of lung disease, and normal spirometry with an FEV1 and FVC within the predicted range. All participants provided written informed consent. This study was approved by the Human Research Ethics Committee, Princess Alexandra Hospital, Brisbane, Australia (EC00167).

\section{Clinical definitions}

Stable COPD was defined as no change in exercise tolerance, sputum colour or volume in the previous 6 weeks, and no oral corticosteroid use or increased treatment above maintenance in the preceding 2 weeks. AECOPD was defined as per ATS/ERS criteria as two out of three of increased sputum production, increased dyspnoea or change in sputum colour [2, 21]. No limitations were placed on medications used for AECOPD by treating clinicians. AECOPD patients returned to the outpatient clinic after completing treatment with antibiotics and/ or oral steroids.

\section{Assessment}

Patients completed a questionnaire documenting current and prior smoking, history of respiratory and cardiovascular disease, diabetes and use of medications. Post-bronchodilator spirometry was performed according to ATS criteria and FEV1, FVC and FEV1/FVC ratio were recorded as \% pred. One fasting blood sample was obtained from stable COPD patients, while AECOPD patients had two blood samples, one within $48 \mathrm{~h}$ of hospital admission, and a second convalescent sample when clinically stable.

\section{Measurement of SRAGE, SAA and CRP}

As sRAGE may vary in relation to diabetes and hyperlipidaemia [23], fasting lipids, glucose and CRP were analysed by the hospital pathology service using commercial auto-analysers. Plasma was stored at $-20^{\circ} \mathrm{C}$ for later batch analyses of sRAGE, SAA and S100A12 levels. sRAGE (R\&D Systems, Minneapolis, MN, USA), SAA (Anogen, Mississauga, ON, Canada) and S100A12 levels (Cyclex, Nagano, Japan) were analysed by ELISA according to manufacturer's directions. Detection limits for the sRAGE, SAA and S100A12 assays were $19.5 \mathrm{pg} \cdot \mathrm{mL}^{-1}$, $1.25 \mathrm{ng} \cdot \mathrm{mL}^{-1}$ and $78 \mathrm{pg} \cdot \mathrm{mL}^{-1}$, respectively. The sRAGE intraassay and interassay coefficients of variation are reported to be $<6 \%$ and $<8 \%$, respectively [11].

\section{Statistical analysis}

SPSS (SPSS Inc., Chicago, IL, USA) was used for data analysis and group differences were assessed by Mann-Whitney U-test, Fischer's exact test or ANCOVA. ANCOVA controls for the influence of confounding variables on a test variable that is being compared between two subject groups. Paired samples were compared by Wilcoxon signed-rank test. Correlations between variables were determined using Spearman's rho. For simple and multiple linear regression analysis, variables were examined for normal distribution and if necessary were natural log-transformed before further analysis. Simple linear regression analysis was initially used to evaluate the relationship between variables and sRAGE. Those variables in which $\mathrm{p}<0.1$ were then subjected to multiple linear regression analysis followed by interaction and residual analyses. Simple and multivariate logistic regressions were used to assess the relationship between plasma levels of sRAGE, CRP or SAA and COPD.

\section{RESULTS}

\section{Subject characteristics}

Between February 2008 and March 2009, 61 patients with COPD and 42 healthy controls were recruited into this casecontrol study. The characteristics of the subjects are outlined in table 1, and additional clinical information is provided in the online supplementary material in table I. As a group, the COPD patients were older than the healthy controls, were more likely to be male and had a lower body mass index (BMI). 


\begin{tabular}{lccc} 
TABLE 1 & Subject characteristics & & \\
& $\begin{array}{c}\text { Healthy } \\
\text { controls }\end{array}$ & $\begin{array}{c}\text { Stable COPD } \\
\text { patients }\end{array}$ & p-value \\
\hline Subjects n & 42 & 61 & \\
Age yrs & $61.6(57.6-71.3)$ & $70.0(63.0-74.3)$ & 0.009 \\
Males & $17(40)$ & $37(61)$ & 0.048 \\
BMI & $27.1(25.5-31.2)$ & $25.7(21.8-29.9)$ & 0.005 \\
Ever smoker & $14(33)$ & $61(100)$ & $<0.001$ \\
Current smoker & $0(0)$ & $17(28)$ & $<0.001$ \\
Smoking pack-yrs & $9.5(5-22)$ & $52.5(36-75)$ & 0.001 \\
FEV 1 \% pred & $95(87-105)$ & $37(23-49)$ & $<0.001$ \\
Inhaled steroids & $0(0)$ & $45(74)$ & $<0.001$ \\
\hline
\end{tabular}

Data are presented as median (interquartile range) and $n(\%)$, unless otherwise stated. COPD: chronic obstructive pulmonary disease; BMI: body mass index; FEV1: forced expiratory volume in $1 \mathrm{~s} ; \%$ pred: \% predicted.

As expected, more of the COPD patients had smoked and they demonstrated lower FEV1 values than the healthy controls $(\mathrm{p}<0.001$; table 1$) .74 \%$ of the COPD patients were prescribed inhaled steroids. Based on the GOLD criteria, 14 patients had moderate COPD, 28 had severe COPD and 19 had very severe COPD (supplementary table I). The COPD group reported a history of cardiovascular disease more frequently than control subjects $(p=0.014)$, while hypertension, hyperlipidaemia and diabetes were present to a similar extent in both COPD and healthy control groups (supplementary table I). There were no significant inter-group differences in relation to fasting concentrations of glucose, low-density lipoprotein (LDL) cholesterol, high-density lipoprotein (HDL) cholesterol and triglycerides, or to the use of lipid-lowering medications (supplementary table I).

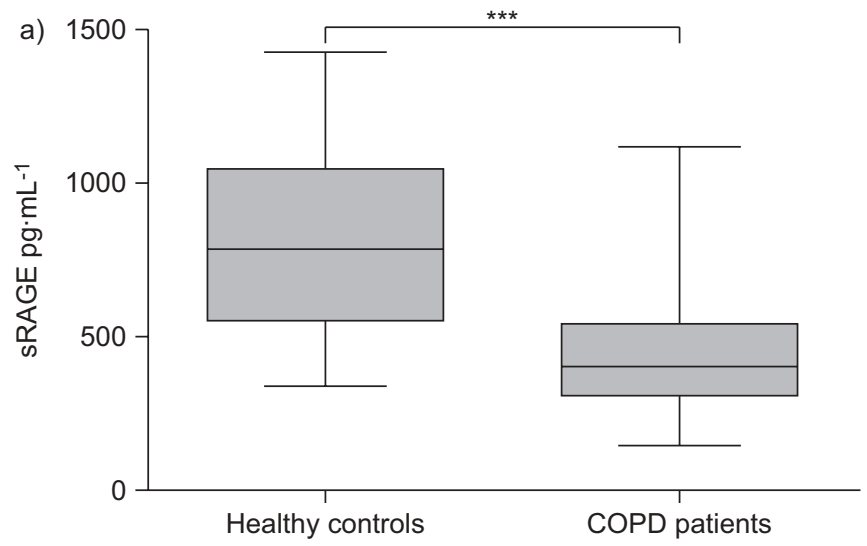

\section{Plasma sRAGE in stable COPD}

Median plasma sRAGE was significantly lower in stable COPD patients $\left(400.2 \mathrm{pg} \cdot \mathrm{mL}^{-1}\right)$ than in healthy control subjects $\left(783.3 \mathrm{pg} \cdot \mathrm{mL}^{-1} ; \mathrm{p}<0.001\right)$, as shown in figure 1a. Even when the data was corrected for age and sex using ANCOVA, plasma sRAGE levels remained significantly lower in COPD than in healthy control subjects $(p<0.001)$. Given that age and sex were not identical between the two groups, a subset of 28 pairs of patients and healthy control subjects matched for these characteristics were blindly selected by an independent observer with no knowledge of the sRAGE results. In this subset, plasma sRAGE was still significantly lower in the COPD patients than in the control subjects (supplementary fig. 1), confirming the findings of the ANCOVA analysis. Similarly, when subjects were separated on the basis of smoking status, plasma sRAGE remained significantly lower in the COPD patients compared to controls, irrespective of current smoking status (fig. 1b). A significant correlation was noted between FEV1 and plasma sRAGE $\left(\mathrm{r}^{2}=0.530, \mathrm{p}<0.001\right)$ as shown in figure 2 . Note that this figure contains data for all 103 subjects in the study, both COPD patients and healthy control subjects. When the analysis was restricted to COPD patients alone, a nonsignificant trend for lower sRAGE levels with lower FEV1 remained $\left(r^{2}=0.224, p=0.082\right)$. Carbon monoxide gas transfer was measured in 36 (59\%) of the COPD patients in the study, but neither the diffusing capacity of the lung for carbon monoxide $(D \mathrm{~L}, \mathrm{CO})$ nor the diffusing capacity per unit alveolar volume $(\mathrm{KCO})$ varied significantly in relation to plasma sRAGE (data not shown).

\section{Other inflammatory markers in stable COPD}

Median serum CRP was significantly higher in stable COPD patients $\left(5.0 \mathrm{mg} \cdot \mathrm{L}^{-1}\right)$ than in healthy control subjects $\left(2.0 \mathrm{mg} \cdot \mathrm{L}^{-1}\right.$; $\mathrm{p}<0.001$ ), as shown in figure 3. Plasma concentrations of the RAGE ligand SAA were also significantly higher in stable COPD patients $\left(7.9 \mu \mathrm{g} \cdot \mathrm{mL}^{-1}\right)$ than in healthy control subjects

b)

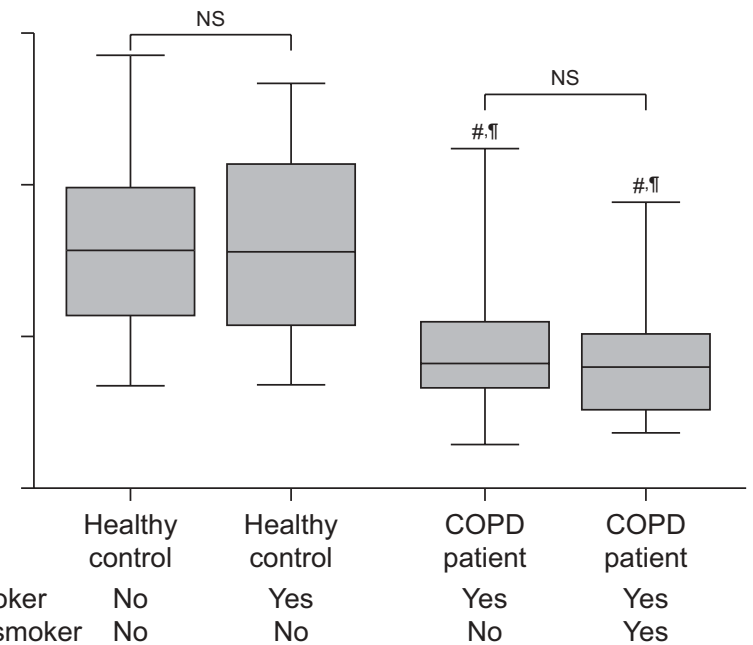

FIGURE 1. Plasma soluble receptor for advanced glycation end-products (SRAGE) in healthy subjects and chronic obstructive pulmonary disease (COPD) patients. a) sRAGE was measured in plasma from healthy control subjects $(n=42)$ and stable COPD patients $(n=61)$. ***: $p<0.001$. b) Healthy control subjects were divided into those who have never smoked $(n=28)$, and those with a history of smoking $(n=14)$. Stable COPD patients were divided into ex-smokers $(n=44)$ and current smokers $(n=17)$. The data was first analysed by the non-parametric Kruskal-Wallis test for multiple groups with $p<0.001$, allowing multiple group comparisons to be assessed by Mann-Whitney tests. Box and whisker plots represent medians, interquartile ranges and range. ${ }^{*}: p<0.001$ versus healthy never-smokers; ${ }^{\circ}: p<0.001$ versus healthy former smokers. NS: nonsignificant. 


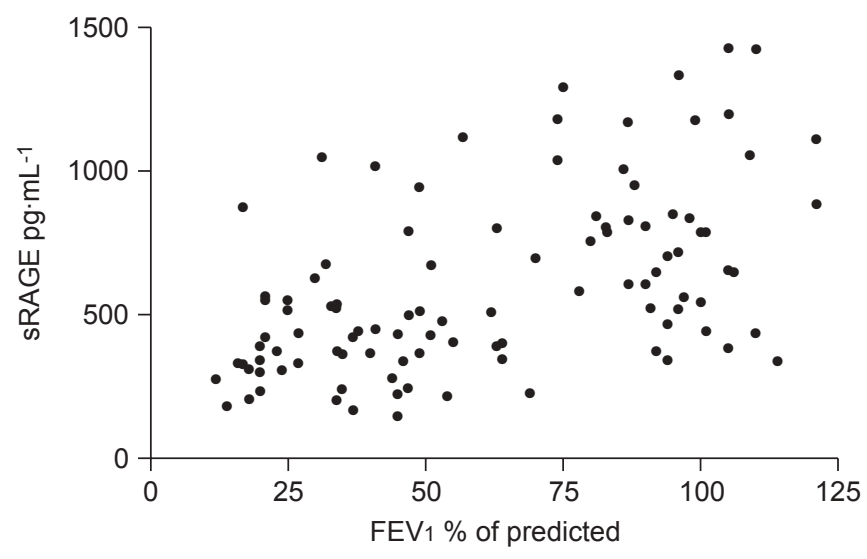

FIGURE 2. Correlation between plasma soluble receptor for advanced glycation end-products (SRAGE) and forced expiratory volume in $1 \mathrm{~s}$ (FEV1) \% of predicted. Plasma sRAGE was correlated with FEV $1 \%$ of pred using Spearman's rho in the total cohort $(n=103)$ with $r^{2}=0.530$ and $p<0.001$

$\left(4.1 \mu \mathrm{g} \cdot \mathrm{mL}^{-1} ; \mathrm{p}<0.015\right)$, as shown in figure 3. Significant inverse correlations were noted between FEV1 and CRP $\left(r^{2}=-0.333\right.$, $p=0.001)$ and between FEV1 and SAA $\left(r^{2}=-0.251, p=0.011\right.$; data not shown). Plasma concentrations of the RAGE ligand S100A12 were similar in COPD patients and control subjects (supplementary fig. 2).

\section{Variables associated with plasma sRAGE and FEV 1}

Simple univariate linear regression analysis of data from all study subjects showed that plasma sRAGE was lower in relation to having COPD, to having ever smoked, to a history of cardiovascular disease and using inhaled steroids, and inversely proportional to the total pack-yrs of cigarette exposure, plasma $\mathrm{CRP}$ and plasma SAA, and directly proportional to FEV1 $(p<0.05$, table 2$)$. These variables were then used in a multivariate linear regression analysis, with the exception of FEV1 and pack-yrs of smoke exposure which were removed from the model as they showed a very high degree of collinearity with COPD and ever smoking, respectively. Plasma sRAGE was not linearly associated with age, sex, BMI, history of diabetes, hypertension and hyperlipidaemia and fasting levels of triglycerides, and LDL and HDL cholesterol. Multiple linear regression with plasma sRAGE as the dependent variable showed that COPD was independently and negatively associated with sRAGE $(\mathrm{p}<0.001$; table 2$)$. When all three inflammatory markers (sRAGE, CRP and SAA) were evaluated via multiple logistic regression analysis with COPD as the dependent variable, only sRAGE was a good predictor of COPD $(\mathrm{p}<0.001)$ (table 3$)$. Plasma sRAGE was similar in COPD patients with a history of cardiovascular disease (median 427.6, interquartile range (IQR) 309.1-623.7; $\mathrm{n}=22$ ) and in COPD patients without a history of cardiovascular disease (median 399.0, IQR 326.0-516.6; $n=32$; $\mathrm{p}=0.718)$.

\section{Plasma sRAGE and other inflammatory markers in AECOPD}

During the study, 24 patients in the COPD group had paired blood samples taken during an AECOPD requiring hospitalisation, and again when they were clinically stable (median 8 weeks later). Figure 4 shows that median plasma sRAGE was lower during the acute exacerbation $\left(252.0 \mathrm{pg} \cdot \mathrm{mL}^{-1}\right)$ than during
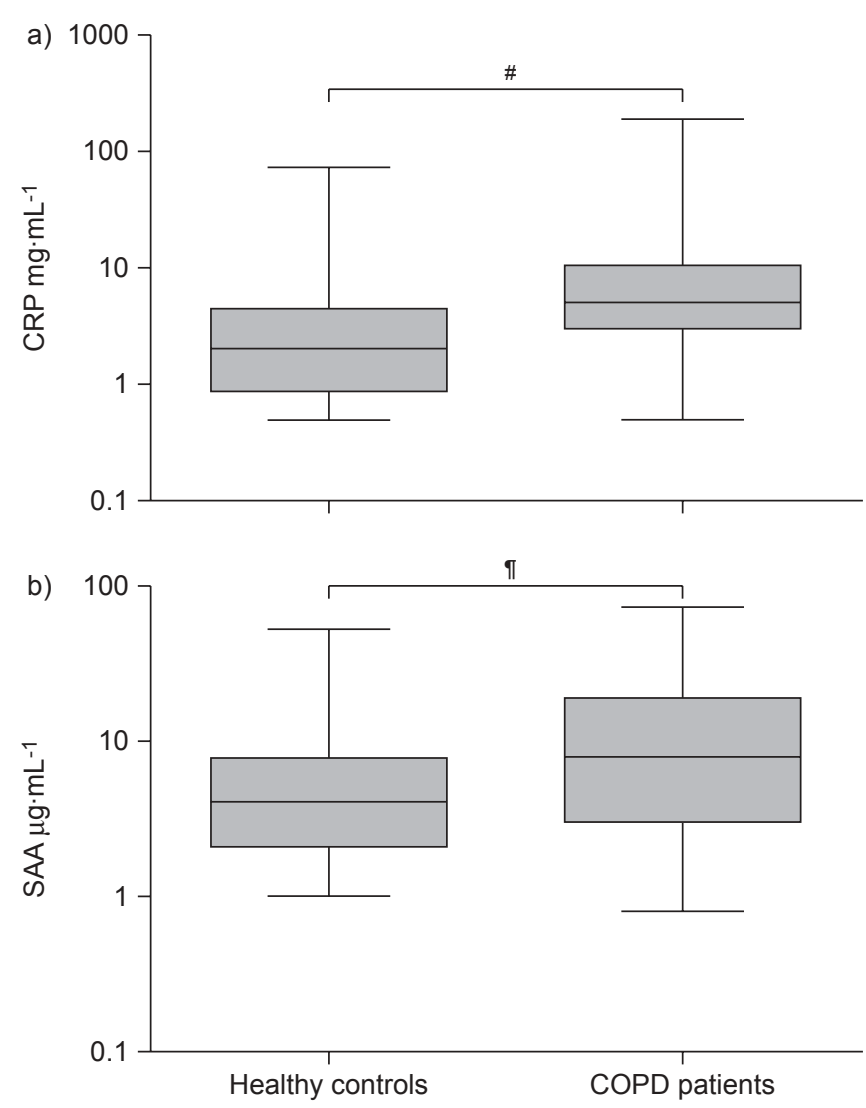

FIGURE 3. a) C-reactive protein (CRP) and b) serum amyloid $A(S A A)$ in healthy subjects and chronic obstructive pulmonary disease (COPD) patients. Serum CRP and plasma SAA from healthy control subjects $(n=42)$ and stable COPD patients $(n=61)$. Box and whisker plots represent medians, interquartile ranges and range. ${ }^{*}: p=0.001 ; ": p=0.015$.

convalescence $\left(344.7 \mathrm{pg} \cdot \mathrm{mL}^{-1} ; \mathrm{p}<0.001\right)$. In contrast, plasma CRP (median $12.0 \mathrm{mg} \cdot \mathrm{L}^{-1}$ versus $6.5 \mathrm{mg} \cdot \mathrm{L}^{-1}, \mathrm{p}=0.007$ ) and SAA $\left(63.5 \mu \mathrm{g} \cdot \mathrm{mL}^{-1}\right.$ versus $\left.8.8 \mu \mathrm{g} \cdot \mathrm{mL}^{-1}, \mathrm{p}<0.001\right)$ were higher during the acute exacerbation than during convalescence.

\section{DISCUSSION}

The key findings to emerge from this study are that plasma concentrations of the anti-inflammatory molecule sRAGE are reduced in patients with stable COPD, relative to healthy control subjects, and that plasma sRAGE is correlated with the severity of airflow obstruction as measured by FEV1. Furthermore, plasma sRAGE was even lower during AECOPD than in stable COPD. To the best of our knowledge this is the first study that has investigated plasma sRAGE in COPD.

An emerging body of literature has shown similar links between reduced levels of sRAGE and other chronic inflammatory diseases, such as rheumatoid arthritis and coronary atherosclerosis [11, 12]. However, it seems unlikely that our findings of reduced plasma sRAGE in COPD can be attributed to cardiac disease or diabetes, as neither of these were independent predictors of sRAGE levels in our cohort by multivariate linear regression analysis (table 2). Furthermore, the strong statistical association between reduced sRAGE and COPD remained even when we corrected for a history of 


\begin{tabular}{|c|c|c|c|c|c|c|}
\hline \multirow{2}{*}{$\begin{array}{l}\text { TABLE } 2 \\
\text { Analysis }\end{array}$} & \multicolumn{6}{|c|}{$\begin{array}{l}\text { Linear regression analysis of all } 103 \text { subjects with } \\
\text { plasma soluble receptor for advanced glycation } \\
\text { end-products (sRAGE) levels as the dependent } \\
\text { variable }\end{array}$} \\
\hline & & $\beta$ & SE & t-test & $p$-value & $95 \% \mathrm{Cl}$ for $\beta$ \\
\hline \multicolumn{7}{|l|}{ Univariate } \\
\hline COPD & & -0.604 & 0.088 & -6.831 & $<0.001$ & $-0.780--0.429$ \\
\hline FEV $1 \%$ pre & & 0.457 & 0.073 & 6.235 & $<0.001$ & $0.311-0.602$ \\
\hline Ever smoke & & -0.486 & 0.108 & -4.508 & $<0.001$ & $-0.700--0.272$ \\
\hline $\begin{array}{c}\text { Cigarette ex } \\
\text { pack-yrs }\end{array}$ & osure & -0.006 & 0.001 & -4.806 & $<0.001$ & $-0.009--0.004$ \\
\hline $\begin{array}{c}\text { History of co } \\
\text { disease }\end{array}$ & diovascular & -0.232 & 0.116 & -2.001 & 0.048 & $-0.463--0.002$ \\
\hline $\begin{array}{l}\text { Use of lipid- } \\
\text { medicatio }\end{array}$ & owering & -0.152 & 0.081 & -1.875 & 0.064 & $-0.313--0.009$ \\
\hline Use of inha & d steroids & -0.541 & 0.091 & -5.928 & $<0.001$ & $-0.722--0.360$ \\
\hline $\mathrm{CRP}^{\#}$ & & -0.149 & 0.041 & -3.629 & $<0.001$ & $-0.230--0.067$ \\
\hline $\mathrm{SAA}^{\#}$ & & -0.134 & 0.063 & -2.131 & 0.037 & $-0.259--0.009$ \\
\hline \multicolumn{7}{|l|}{ Multivariate } \\
\hline COPD & & -0.592 & 0.105 & -5.616 & $<0.001$ & $-0.803--0.382$ \\
\hline
\end{tabular}

COPD: chronic obstructive pulmonary disease; FEV1: forced expiratory volume in $1 \mathrm{~s}$; \% pred: \% predicted; CRP: C-reactive protein; SAA: serum amyloid A. \#: variable was natural log-transformed, as was sRAGE. Only variables with $p<0.01$ are shown in the table. $r^{2}$ of the multivariate linear regression $=0.327$. Adjusted $r^{2}=0.316$.

cardiac disease and diabetes using an ANCOVA. Very few patients in the study had rheumatoid arthritis.

In relation to lung disease, reduced sRAGE expression has been reported by two independent groups in idiopathic pulmonary fibrosis $[14,18]$, though the relationships between lung function and sRAGE were not examined in these studies. In contrast, prior studies of acute lung injury have reported high circulating sRAGE $[16,17]$. The disparity between these studies may reflect differing mechanisms of lung injury in diverse lung diseases. FERHANI et al. [19] recently reported that membrane RAGE is overexpressed in the airway epithelium and smooth muscle of COPD patients, though some overlap was noted between COPD patients and healthy smokers. The authors did not measure sRAGE in that study. We may speculate that high expression of membrane RAGE in COPD lungs might be associated with low circulating concentrations of sRAGE, and this will be an important issue for future studies.

As both membrane and sRAGE appear to have a protective role in a number of animal models involving the lungs and other organs $[9,10,14,18]$, the concept has emerged that decreased circulating sRAGE could be a marker of deficient inflammatory control. If this notion is correct, smokers with decreased blood levels of sRAGE might be predisposed to more lung inflammation and tissue damage, and therefore more likely to develop COPD. Our results suggest that smoking per se does not lead to reduced circulating sRAGE, as plasma sRAGE was similar in healthy control subjects who had ever smoked in comparison with those who had never

\begin{tabular}{ccccc} 
TABLE 3 & $\begin{array}{l}\text { Logistic regression analysis of inflammatory } \\
\text { markers in all } 103 \text { subjects with chronic } \\
\text { obstructive pulmonary disease as the dependent } \\
\text { variable }\end{array}$ \\
Analysis & $\boldsymbol{\beta}$ & SE & p-value & OR (95\% CI for $\boldsymbol{\beta})$ \\
\hline $\begin{array}{c}\text { Univariate } \\
\text { SRAGE }^{\#}\end{array}$ & -3.0701 & 0.6261 & $<0.001$ & $0.0464(0.0136-0.1583)$ \\
SAA $^{*}$ & 0.5378 & 0.2106 & 0.011 & $1.7123(1.1333-2.5872)$ \\
CRP $^{\#}$ & 0.6116 & 0.1946 & 0.0017 & $1.8434(1.2589-2.6994)$ \\
Multivariate $^{\text {sRAGE }}$ & -2.7626 & 0.6254 & $<0.001$ & $0.0631(0.0185-0.2150)$ \\
\hline
\end{tabular}

SRAGE: soluble receptor for advanced glycation end-products; SAA: serum amyloid A; CRP: C-reactive protein. ${ }^{*}$ : variable was natural log-transformed.

smoked (fig. 1b), and significantly higher $(p<0.001)$ than in COPD patients. Although the univariate analysis suggested a relationship between plasma sRAGE and previous smoking history (table 2), multiple linear regression analysis indicated that a prior smoking history was not an independent predictor of plasma sRAGE. This suggests that other mechanisms lead to reduced sRAGE.

Functional polymorphisms in the AGER gene that encodes RAGE are associated with rheumatoid arthritis [24, 25], and it was recently reported that SRAGE levels were strongly associated with carriage of the rheumatoid arthritis-associated RAGE $82 \mathrm{~S}$ polymorphism [24]. Large community-based, genome-wide association studies have recently reported that single nucleotide polymorphisms in the AGER gene are associated with a reduced FEV1/FVC ratio indicative of airflow obstruction [26, 27]. Such polymorphisms may alter the extent to which membrane bound RAGE is cleaved to sRAGE, though this remains to be proven. Alternatively, reduced circulating sRAGE levels may identify individuals exposed to high levels of RAGE ligands and other inflammatory mediators, such that sRAGE is consumed or "mopped-up" in the lungs or circulation. Given that alveolar cells are a major source of RAGE in the lungs a further possible explanation is that low sRAGE levels are a direct consequence of alveolar cell loss due to emphysema. However, neither DL,CO nor KCO varied significantly in relation to plasma sRAGE.

AECOPD were associated with further reductions in plasma sRAGE and high serum CRP concentrations (fig. 4), and both markers returned towards baseline at convalescence. COPD exacerbations are frequently triggered by bacterial or viral infections, and are associated with pulmonary inflammation, hypoxia and release of cytokines and pro-inflammatory mediators [6]. The low plasma sRAGE concentrations seen in AECOPD may be a direct result of release of pro-inflammatory mediators. Experimental studies indicate that tumour necrosis factor (TNF)- $\alpha$ for example can down-regulate RAGE expression in lung epithelial cells and fibroblasts [18], and while we did not measure TNF- $\alpha$ in the current study, others have demonstrated that TNF- $\alpha$ levels are increased during AECOPD [28]. Hypoxia can stimulate the generation of RAGE ligands [29], and this is an additional mechanism by which AECOPD might induce changes in plasma sRAGE. 

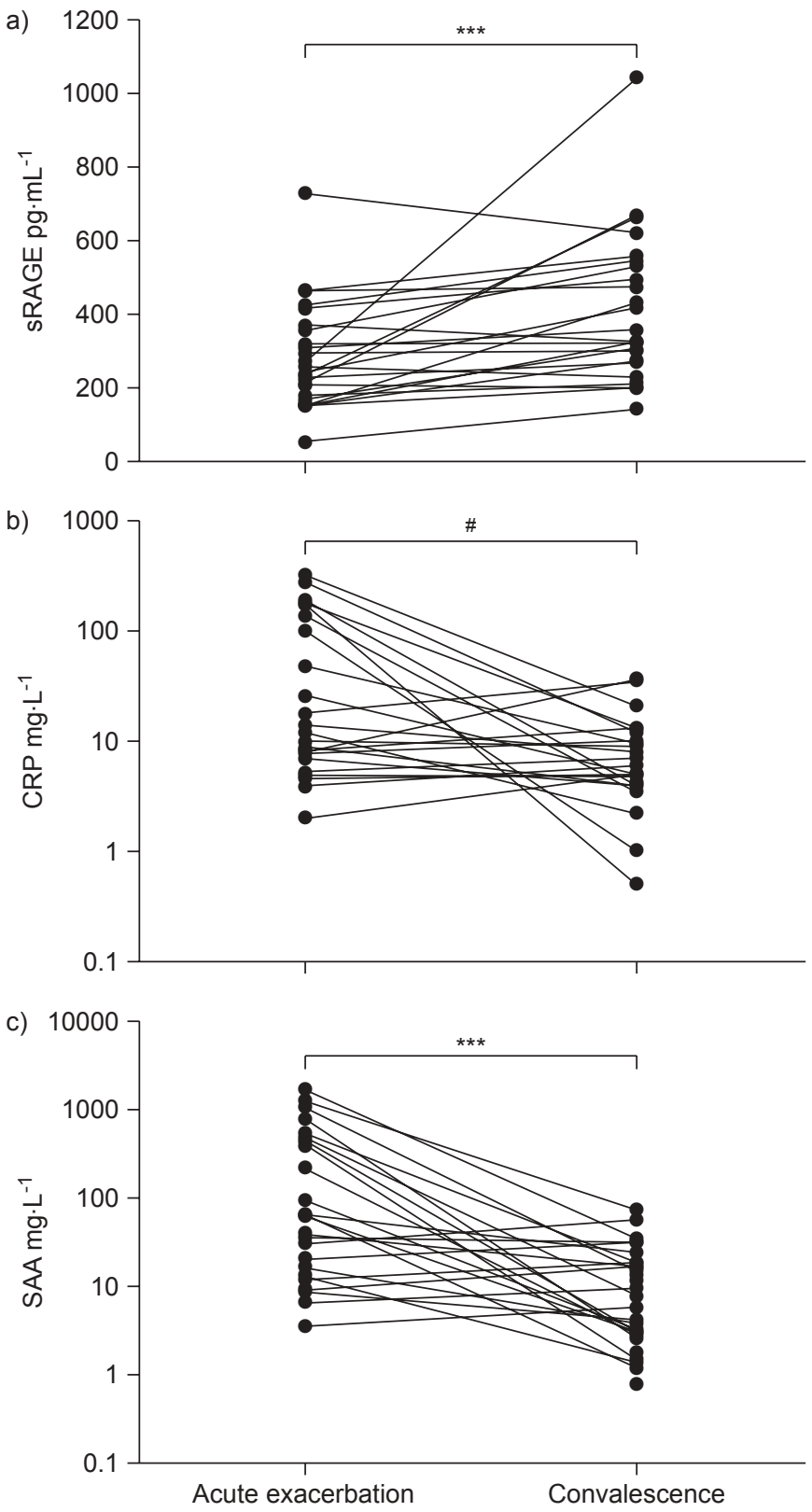

FIGURE 4. a) Soluble receptor for advanced glycation end-products (sRAGE), b) C-reactive protein (CRP) and c) serum amyloid A (SAA) during an acute exacerbation and at convalescence. Median circulating concentrations of SRAGE, CRP and SAA during an acute exacerbation were $252.0 \mathrm{pg} \cdot \mathrm{mL}^{-1}, 12.0 \mathrm{mg} \cdot \mathrm{L}^{-1}$ and $63.5 \mu \mathrm{g} \cdot \mathrm{mL}^{-1}$ compared to $344.7 \mathrm{pg} \cdot \mathrm{mL}^{-1}, 6.5 \mathrm{mg} \cdot \mathrm{L}^{-1}$ and $8.8 \mu \mathrm{g} \cdot \mathrm{mL}^{-1}$ at convalescence, respectively $(n=24)$. ${ }^{* *}: p<0.001 ;{ }^{*}: p=0.007$.

There are a number of limitations to the current study that should be borne in mind. Despite our best efforts it was not possible to achieve perfect matching of cases and controls. However, when the analysis was adjusted for age and sex using ANCOVA, there remained a significant reduction in sRAGE in the COPD group compared to the healthy controls. While COPD patients and healthy controls exhibited differences with regard to smoking, the use of inhaled steroids and prevalence of cardiovascular disease, multivariate linear regression did not identify these as being predictors of plasma sRAGE. In addition, we measured sRAGE in plasma rather than lung because of the practical issues associated with sampling BAL in a study of this size, and because the sRAGE ELISA assay has not been validated for use in sputum. However, it will be important for future studies to compare the blood and lung expression of sRAGE, membrane RAGE and RAGE ligands in COPD. Finally, we cannot exclude an effect of systemic corticosteroids on plasma sRAGE concentration in AECOPD. All patients with an AECOPD had blood sampling performed within $48 \mathrm{~h}$ of admission to hospital, and had therefore been treated with at least one dose of oral prednisolone. Though this might have biased the sRAGE data to some extent, we believe that any effects were probably modest, given that the serum CRP at the same time-point was high (fig. 4).

Even though plasma sRAGE was the prime focus of this study, we also measured three other inflammatory markers in the circulation. CRP is an acute-phase protein produced in the liver and our finding that CRP was increased in stable COPD relative to healthy subjects is consistent with previous reports $[6,30,31]$. Serum CRP was inversely correlated with both sRAGE levels and FEV1 in the current study, and we recently noted a similar inverse correlation with sRAGE and CRP in rheumatoid arthritis [25]. SAA is also an acute phase protein, but is a known RAGE ligand and can be produced in the lung $[32,33]$. Our data shows for the first time that plasma SAA is increased in stable COPD relative to healthy subjects. It was recently reported that SAA concentrations were similar in stable COPD and healthy subjects [20], though that study contained only a modest number of normal subjects and may have been slightly underpowered. It is important to emphasise that COPD was independently correlated with sRAGE, while plasma SAA did not predict SRAGE (table 2), suggesting a relatively direct link between COPD and sRAGE, rather than an indirect mechanism involving SAA. In contrast, plasma concentrations of the RAGE ligand S100A12 were similar in COPD patients and control subjects, so it is possible that different ligands are responsible for activation of RAGE in different disease states.

The need for better biomarkers in COPD is widely acknowledged [34, 35]. Currently, CRP and fibrinogen are accepted biomarkers, but our results suggest that sRAGE is potentially a better predictor of COPD compared to CRP (table 3). This pilot study suggests that sRAGE may have a novel use as a biomarker in COPD, warranting further investigation with large prospective studies.

In conclusion, we have demonstrated plasma sRAGE is reduced in patients with COPD, and that sRAGE is strongly and independently associated with the severity of airflow obstruction as measured by FEV1. These findings imply that sRAGE may play a role in systemic inflammation in COPD and that sRAGE has promise as a potential biomarker in COPD. Future longitudinal studies will be needed to ascertain whether sRAGE levels decline further as the disease progresses and to determine whether sRAGE has utility in predicting COPD exacerbations, comorbidities and mortality in COPD.

\section{SUPPORT STATEMENT}

Funding was provided by the National Health \& Medical Research Council of Australia and Princess Alexandra Hospital Foundation. 


\section{STATEMENT OF INTEREST}

None declared.

\section{ACKNOWLEDGEMENTS}

We would like to thank Y-S. Chen (Diamantina Institute, The University of Queensland, Brisbane, Australia) for his helpful insights.

\section{REFERENCES}

1 Lopez AD, Shibuya K, Rao C, et al. Chronic obstructive pulmonary disease: current burden and future projections. Eur Respir J 2006; 27: 397-412.

2 Rabe KF, Hurd S, Anzueto A, et al. Global strategy for the diagnosis, management, and prevention of chronic obstructive pulmonary disease: GOLD executive summary. Am J Respir Crit Care Med 2007; 176: 532-555.

3 Agusti A. Systemic effects of chronic obstructive pulmonary disease: what we know and what we don't know (but should). Proc Am Thorac Soc 2007; 4: 522-525.

4 Gan WQ, Man SF, Senthilselvan A, et al. Association between chronic obstructive pulmonary disease and systemic inflammation: a systematic review and a meta-analysis. Thorax 2004; 59: 574-580.

5 Sin DD, Man SF. Why are patients with chronic obstructive pulmonary disease at increased risk of cardiovascular diseases? The potential role of systemic inflammation in chronic obstructive pulmonary disease. Circulation 2003; 107: 1514-1519.

6 Pinto-Plata V, Toso J, Lee K, et al. Profiling serum biomarkers in patients with COPD: associations with clinical parameters. Thorax 2007; 62: 595-601.

7 Bierhaus A, Humpert PM, Morcos M, et al. Understanding RAGE, the receptor for advanced glycation end products. J Mol Med 2005; 83: 876-886.

8 Schmidt AM, Yan SD, Yan SF, et al. The multiligand receptor RAGE as a progression factor amplifying immune and inflammatory responses. J Clin Invest 2001; 108: 949-955.

9 Hofmann MA, Drury S, Hudson BI, et al. RAGE and arthritis: the G82S polymorphism amplifies the inflammatory response. Genes Immun 2002; 3: 123-135.

10 Park L, Raman KG, Lee KJ, et al. Suppression of accelerated diabetic atherosclerosis by the soluble receptor for advanced glycation endproducts. Nat Med 1998; 4: 1025-1031.

11 Falcone C, Emanuele E, D'Angelo A, et al. Plasma levels of soluble receptor for advanced glycation end products and coronary artery disease in nondiabetic men. Arterioscler Thromb Vasc Biol 2005; 25: 1032-1037.

12 Pullerits R, Bokarewa M, Dahlberg L, et al. Decreased levels of soluble receptor for advanced glycation end products in patients with rheumatoid arthritis indicating deficient inflammatory control. Arthritis Res Ther 2005; 7: R817-R824.

13 Cheng C, Tsuneyama K, Kominami R, et al. Expression profiling of endogenous secretory receptor for advanced glycation end products in human organs. Mod Pathol 2005; 18: 1385-1396.

14 Englert JM, Hanford LE, Kaminski N, et al. A role for the receptor for advanced glycation end products in idiopathic pulmonary fibrosis. Am J Pathol 2008; 172: 583-591.

15 Shirasawa M, Fujiwara N, Hirabayashi S, et al. Receptor for advanced glycation end-products is a marker of type I lung alveolar cells. Genes Cells 2004; 9: 165-174.

16 Uchida T, Shirasawa M, Ware LB, et al. Receptor for advanced glycation end-products is a marker of type I cell injury in acute lung injury. Am J Respir Crit Care Med 2006; 173: 1008-1015.
17 Calfee CS, Ware LB, Eisner MD, et al. Plasma receptor for advanced glycation end products and clinical outcomes in acute lung injury. Thorax 2008; 63: 1083-1089.

18 Queisser MA, Kouri FM, Konigshoff M, et al. Loss of RAGE in pulmonary fibrosis: molecular relations to functional changes in pulmonary cell types. Am J Respir Cell Mol Biol 2008; 39: 337-345.

19 Ferhani N, Letuve S, Kozhich A, et al. Expression of high-mobility group box 1 and of receptor for advanced glycation end products in COPD. Am J Respir Crit Care Med 2010; 181: 917-927.

20 Bozinovski S, Hutchinson A, Thompson M, et al. Serum amyloid A is a biomarker of acute exacerbations of chronic obstructive pulmonary disease. Am J Respir Crit Care Med 2008; 177: 269-278.

21 Celli BR, MacNee W, Agusti A, et al. Standards for the diagnosis and treatment of patients with COPD: a summary of the ATS/ERS position paper. Eur Respir J 2004; 23: 932-946.

22 Kalousova M, Hodkova M, Kazderova M, et al. Soluble receptor for advanced glycation end products in patients with decreased renal function. Am J Kidney Dis 2006; 47: 406-411.

23 Nakamura K, Yamagishi S, Adachi H, et al. Elevation of soluble form of receptor for advanced glycation end products (sRAGE) in diabetic subjects with coronary artery disease. Diabetes Metab Res Rev 2007; 23: 368-371.

24 Carroll L, Frazer IH, Turner M, et al. Receptor for advanced glycation end products Glycine 82 Serine polymorphism and risk of cardiovascular events in rheumatoid arthritis. Arthritis Res Ther 2007; 9: R39.

25 Chen YS, Yan W, Geczy CL, et al. Serum levels of soluble receptor for advanced glycation end products and of S100 proteins are associated with inflammatory, autoantibody, and classical risk markers of joint and vascular damage in rheumatoid arthritis. Arthritis Res Ther 2009; 11: R39.

26 Hancock DB, Eijgelsheim M, Wilk JB, et al. Meta-analyses of genome-wide association studies identify multiple loci associated with pulmonary function. Nat Genet 2010; 42: 45-52.

27 Repapi E, Sayers I, Wain LV, et al. Genome-wide association study identifies five loci associated with lung function. Nat Genet 2010; 42: 36-44.

28 Sethi S, Wrona C, Eschberger K, et al. Inflammatory profile of new bacterial strain exacerbations of chronic obstructive pulmonary disease. Am J Respir Crit Care Med 2008; 177: 491-497.

29 Chang JS, Wendt $\mathrm{T}, \mathrm{Qu} \mathrm{W}$, et al. Oxygen deprivation triggers upregulation of early growth response- 1 by the receptor for advanced glycation end products. Circ Res 2008; 102: 905-913.

30 de Torres JP, Cordoba-Lanus E, López-Aguilar C, et al. C-reactive protein levels and clinically important predictive outcomes in stable COPD patients. Eur Respir J 2006; 27: 902-907.

31 Gan WQ, Man SF, Sin DD. The interactions between cigarette smoking and reduced lung function on systemic inflammation. Chest 2005; 127: 558-564.

32 Merkel D, Rist W, Seither P, et al. Proteomic study of human bronchoalveolar lavage fluids from smokers with chronic obstructive pulmonary disease by combining surface-enhanced laser desorption/ionization-mass spectrometry profiling with mass spectrometric protein identification. Proteomics 2005; 5: 2972-2980.

33 Yip TT, Chan JW, Cho WC, et al. Protein chip array profiling analysis in patients with severe acute respiratory syndrome identified serum amyloid A protein as a biomarker potentially useful in monitoring the extent of pneumonia. Clin Chem 2005; 51: 47-55.

34 Cazzola M, MacNee W, Martinez FJ, et al. Outcomes for COPD pharmacological trials: from lung function to biomarkers. Eur Respir J 2008; 31: 416-469.

35 Sin DD, Man SF. Biomarkers in COPD: are we there yet? Chest 2008; 133: 1296-1298. 